\title{
Microscopic calculation of the interaction cross section for stable and unstable nuclei based on the nonrelativistic nucleon-nucleon $t$-matrix
}

\author{
Dao T. Khoa* and Hoang Sy Than \\ Institute for Nuclear Science \& Technique, VAEC, P.O. Box 5T-160, Nghia Do, Hanoi, Vietnam. \\ Tran Hoai Nam \\ Department of Physics, Hanoi University of Natural Sciences, \\ 334 Nguyen Trai Str., Thanh Xuan, Hanoi, Vietnam. \\ Marcella Grasso and Nguyen Van Giai \\ Institut de Physique Nucléaire, IN2P3-CNRS, 91406 Orsay Cedex, France.
}

(Dated: Accepted for publication in Physical Review C)

\begin{abstract}
Fully quantal calculations of the total reaction cross sections $\sigma_{\mathrm{R}}$ and interaction cross sections $\sigma_{\mathrm{I}}$, induced by stable and unstable $\mathrm{He}, \mathrm{Li}, \mathrm{C}$ and $\mathrm{O}$ isotopes on ${ }^{12} \mathrm{C}$ target at $E_{\mathrm{lab}} \approx 0.8$ and 1 $\mathrm{GeV} /$ nucleon have been performed, for the first time, in the distorted wave impulse approximation (DWIA) using the microscopic complex optical potential and inelastic form factors given by the folding model. Realistic nuclear densities for the projectiles and ${ }^{12} \mathrm{C}$ target as well as the complex $t$ matrix parameterization of free nucleon-nucleon interaction by Franey and Love were used as inputs of the folding calculation. Our parameter-free folding + DWIA approach has been shown to give a very good account (within 1-2\%) of the experimental $\sigma_{\text {I }}$ measured at these energies for the stable, strongly bound isotopes. With the antisymmetrization of the dinuclear system properly taken into account, this microscopic approach is shown to be more accurate than the simple optical limit of Glauber model that was widely used to infer the nuclear radii from the measured $\sigma_{\mathrm{I}}$. Therefore, the results obtained for the nuclear radii of neutron-rich isotopes under study can be of interest for further nuclear structure studies.
\end{abstract}

PACS numbers: 24.10.Eq, 24.10.Ht, 24.50.+g, 25.60.Bx, 25.60.Dz

\section{INTRODUCTION}

Since the 80 s of the last century, the radioactive ion beams have been used intensively to measure the total reaction cross sections and interaction cross sections induced by unstable nuclei on stable targets (see a recent review in Ref. [1]) which serve as an important data bank for the determination of nuclear sizes. The discovery of exotic structures of unstable nuclei, such as neutron halos or neutron skins, are among the most fascinating results of this study.

The theoretical tool used dominantly by now to analyze the interaction cross sections measured at energies of several hundred $\mathrm{MeV} /$ nucleon is the Glauber model $[2,3]$ which is based on the eikonal approximation. This approach provides a simple connection between the ground state densities of the two colliding nuclei and the total reaction cross section of the nucleus-nucleus system and has been used, in particular, to deduce the nuclear density parameters for the neutron-rich halo nuclei [4].

In general, the total reaction cross section $\sigma_{\mathrm{R}}$, which measures the loss of flux from the elastic channel, must be calculated from the transmission coefficient $T_{l}$ as

$$
\sigma_{\mathrm{R}}=\frac{\pi}{k^{2}} \sum_{l}(2 l+1) T_{l}
$$

where $k$ is the relative momentum (or wave number). The summation is carried over all partial waves $l$ with $T_{l}$ determined from the elastic $S$-matrix as

$$
T_{l}=1-\left|S_{l}\right|^{2} .
$$

In the standard optical model (OM), the quantal $S$ matrix elements $S_{l}$ are obtained from the solution of the Schrödinger equation for elastic nucleus-nucleus scattering using a complex optical potential. At low energies, the eikonal approximation is less accurate and, instead of Glauber model, the OM should be used to calculate $\sigma_{\mathrm{R}}$ for a reliable comparison with the data. At energies approaching $1 \mathrm{GeV} /$ nucleon region, there are very few elastic scattering data available and the choice of a realistic optical potential becomes technically difficult, especially for unstable nuclei. Perhaps, that is the reason why different versions of Glauber model are widely used to calculate $\sigma_{\mathrm{R}}$ at high energies. Depending on the structure model for the nuclear wave functions used in the calculation, those Glauber model calculations can be divided into two groups: the calculations using a simple optical limit of Glauber model (see Ref. [1] and references therein) and the more advanced approaches where the few-body correlation and/or breakup of a looselybound projectile into a core and valence (halo) nucleons are treated explicitly $[3,5,6]$.

In the present work, we explore the applicability of the standard OM to calculate the total reaction cross section (1) induced by stable and unstable beams at high energies using the microscopic optical potential predicted by

*Electronic address: khoa@vaec.gov.vn 
the folding model. The basic inputs of a folding calculation are the densities of the two colliding nuclei and the effective nucleon-nucleon (NN) interaction [7]. At low energies, a realistic density dependent NN interaction [8] based on the M3Y interaction [9] has been successfully used to calculate the $\alpha$-nucleus and nucleus-nucleus optical potential [10]. This interaction fails, however, to predict the shape of the $\alpha$-nucleus optical potential as the bombarding energy increases to about $340 \mathrm{MeV} /$ nucleon [11]. On the other hand, at incident energies approaching a few hundred $\mathrm{MeV} /$ nucleon the $t$-matrix parameterization of free NN interaction was often used in the folding analysis of proton-nucleus scattering [12, 13]. The use of the $t$-matrix interaction corresponds to the so-called impulse approximation (IA), where the medium modifications of the NN interaction are neglected [14].

In the present folding calculation we adopt a local representation of the free NN $t$-matrix developed by Franey and Love [13] based on the experimental NN phase shifts. The folded optical potentials and inelastic form factors are used further in the distorted wave impulse approximation (DWIA) to calculate $\sigma_{\mathrm{R}}$ and interaction cross section $\sigma_{\mathrm{I}}$, induced by stable and unstable $\mathrm{He}, \mathrm{Li}, \mathrm{C}$, and $\mathrm{O}$ isotopes on ${ }^{12} \mathrm{C}$ target at bombarding energies around 0.8 and $1 \mathrm{GeV} /$ nucleon. Since relativistic effects are significant at high energies, the relativistic kinematics are taken into account properly in both the folding and DWIA calculations. To clarify the adequacy and possible limitation of the present folding model, we also discuss the main approximations made in our approach and compare them with those usually assumed in the Glauber model.

Given the realistic nuclear densities and validity of IA, the folding approach presented below in Sec. II is actually parameter-free and it is necessary to test first the reliability of the model by studying the known stable nuclei before going to study unstable nuclei. Such a procedure is discussed briefly in Sec. III. Then, $\sigma_{\text {I }}$ measured for the neutron-rich $\mathrm{He}, \mathrm{Li}, \mathrm{C}$, and $\mathrm{O}$ isotopes are compared with the results of calculation and the sensitivity of nuclear radii to the calculated $\sigma_{\mathrm{I}}$ is discussed. The discrepancy between $\sigma_{\mathrm{I}}^{\text {calc }}$ and $\sigma_{\mathrm{I}}^{\exp }$ found for some light halo nuclei is discussed in details to indicate possible effects caused by the dynamic few-body correlation. Conclusions are drawn in Sec. IV.

\section{FOLDING MODEL FOR THE COMPLEX NUCLEUS-NUCLEUS OPTICAL POTENTIAL}

The details of the latest double-folding formalism are given in Ref. [10] and we only recall briefly its main features. In general, the projectile-target interaction potential can be evaluated as an energy dependent HartreeFock-type potential of the dinuclear system

$$
U=\sum_{i \in a, j \in A}\left[<i j\left|v_{\mathrm{D}}\right| i j>+<i j\left|v_{\mathrm{EX}}\right| j i>\right]=V_{\mathrm{D}}+V_{\mathrm{EX}},
$$

where the nuclear interaction $V$ is a sum of effective NN interactions $v_{i j}$ between nucleon $i$ in the projectile $a$ and nucleon $j$ in the target $A$. The antisymmetrization of the dinuclear system is done by taking into account the single-nucleon knock-on exchanges.

The direct part of the potential is local (provided that the NN interaction itself is local), and can be written in terms of the one-body densities,

$$
\begin{array}{r}
V_{\mathrm{D}}(E, \boldsymbol{R})=\int \rho_{a}\left(\boldsymbol{r}_{a}\right) \rho_{A}\left(\boldsymbol{r}_{A}\right) v_{\mathrm{D}}(E, \rho, s) d^{3} r_{a} d^{3} r_{A}, \\
\text { where } \boldsymbol{s}=\boldsymbol{r}_{A}-\boldsymbol{r}_{a}+\boldsymbol{R} .
\end{array}
$$

The exchange part is, in general, nonlocal. However, an accurate local approximation can be obtained by treating the relative motion locally as a plane wave [15]

$$
\begin{aligned}
& V_{\mathrm{EX}}(E, \boldsymbol{R})=\int \rho_{a}\left(\boldsymbol{r}_{a}, \boldsymbol{r}_{a}+\boldsymbol{s}\right) \rho_{A}\left(\boldsymbol{r}_{A}, \boldsymbol{r}_{A}-\boldsymbol{s}\right) \\
& \times v_{\mathrm{EX}}(E, \rho, s) \exp \left(\frac{i \boldsymbol{K}(E, \boldsymbol{R}) \cdot \boldsymbol{s}}{M}\right) d^{3} r_{a} d^{3} r_{A} .
\end{aligned}
$$

Here $\rho_{a}\left(\boldsymbol{r}_{a}\right) \equiv \rho_{a}\left(\boldsymbol{r}_{a}, \boldsymbol{r}_{a}\right)$ and $\rho_{a}\left(\boldsymbol{r}_{a}, \boldsymbol{r}_{a}+\boldsymbol{s}\right)$ is the diagonal and nondiagonal parts of the one-body density matrix for the projectile, and similarly for the target. $\boldsymbol{K}(E, \boldsymbol{R})$ is the local momentum of relative motion determined as

$$
K^{2}(E, \boldsymbol{R})=\frac{2 \mu}{\hbar^{2}}\left[E_{\text {c.m. }}-\operatorname{Re} U(E, \boldsymbol{R})-V_{\mathrm{C}}(\boldsymbol{R})\right],
$$

$\mu$ is the reduced mass, $M=a A /(a+A)$ with $a$ and $A$ the mass numbers of the projectile and target, respectively. Here, $U(E, \boldsymbol{R})=V_{\mathrm{D}}(E, \boldsymbol{R})+V_{\mathrm{EX}}(E, \boldsymbol{R})$ and $V_{\mathrm{C}}(\boldsymbol{R})$ are the total nuclear and Coulomb potentials, respectively. More details on the calculation of the direct and exchange potentials (4)-(5) can be found in Refs. [10, 16]. The folding inputs for mass numbers and incident energies were taken as given by the relativistically corrected kinematics [17].

To calculate consistently both the optical potential and inelastic form factor one needs to take into account explicitly the multipole decomposition of the nuclear density that enters the folding calculation [10]

$$
\rho_{J M \rightarrow J^{\prime} M^{\prime}}(\boldsymbol{r})=\sum_{\lambda \mu}<J M \lambda \mu \mid J^{\prime} M^{\prime}>C_{\lambda} \rho_{\lambda}(r)\left[i^{\lambda} Y_{\lambda \mu}(\hat{\boldsymbol{r}})\right]^{*},
$$

where $J M$ and $J^{\prime} M^{\prime}$ are the nuclear spin and its projection in the initial and final states, respectively, and $\rho_{\lambda}(r)$ is the nuclear transition density for the corresponding $2^{\lambda}$-pole excitation. In the present work, we adopt the collective-model Bohr-Mottelson prescription [18] to construct the nuclear transition density for a given excitation in the ${ }^{12} \mathrm{C}$ target as

$$
\rho_{\lambda}(r)=-\delta_{\lambda} \frac{d \rho_{0}(r)}{d r} .
$$

Here $\rho_{0}(r)$ is the total ground state (g.s.) density and $\delta_{\lambda}$ is the deformation length of the $2^{\lambda}$-pole excitation in the ${ }^{12} \mathrm{C}$ target. 
Impulse approximation and the $t$-matrix interaction

If the total spin and isospin are zero for one of the two colliding nuclei $\left({ }^{12} \mathrm{C}\right.$ in our case) only the spin- and isospin-independent components of the central NN forces are necessary for the folding calculation. We discuss now the choice of $v_{\mathrm{D}(\mathrm{EX})}(E, \rho, s)$ for the two bombarding energies of 0.8 and $1 \mathrm{GeV} /$ nucleon. At these high energies, one can adopt the IA which reduces the effective NN interaction approximately to that between the two nucleons in vacuum [14]. Consequently, the microscopic optical potential and inelastic form factors can be obtained by folding the g.s. and transition densities of the two colliding nuclei with an appropriate $t$-matrix parameterization of the free NN interaction.

In the present work, we have chosen the nonrelativistic $t$-matrix interaction which was developed by Franey and Love [13] based on experimental NN phase shifts at bombarding energies of 0.8 and $1 \mathrm{GeV}$. The spin- and isospin-independent direct $\left(v_{\mathrm{D}}\right)$ and exchange $\left(v_{\mathrm{EX}}\right)$ parts of the central NN interaction are then determined from the singlet- and triplet-even (SE,TE) and odd (SO,TO) components of the local $t$-matrix interaction (see Table I of Ref. [13]) as

$v_{\mathrm{D}(\mathrm{EX})}(s)=\frac{k_{a} k_{A}}{16}\left[3 t_{\mathrm{TE}}(s)+3 t_{\mathrm{SE}}(s) \pm 9 t_{\mathrm{TO}}(s) \pm 3 t_{\mathrm{SO}}(s)\right]$.

Here $k_{a}$ and $k_{A}$ are the energy-dependent kinematic modification factors of the $t$-matrix transformation [19] from the NN frame to the $\mathrm{N} a$ and $\mathrm{N} A$ frames, respectively. $k_{a}$ and $k_{A}$ were evaluated using Eq. (19) of Ref. [12]. The explicit, complex strength of the finite-range central $t$ matrix interaction (9) is given in terms of four Yukawas [13]. Since the medium modifications of the NN interaction are neglected in the IA [14], the $t$-matrix interaction (9) does not depend on the nuclear density.

\section{Main steps in the calculation of $\sigma_{\mathrm{I}}$}

With properly chosen g.s. densities for the two colliding nuclei, the elastic scattering cross section and $\sigma_{\mathrm{R}}$ are obtained straightforwardly in the OM calculation using the microscopic optical potential (4)-(6). We recall that the interaction cross section $\sigma_{\mathrm{I}}$ is actually the sum of all particle removal cross sections from the projectile [1] and accounts, therefore, for all processes when the neutron and/or proton number in the projectile is changed. As a result, $\sigma_{\mathrm{I}}$ must be smaller than the total reaction cross section $\sigma_{\mathrm{R}}$ which includes also the cross section of inelastic scattering to excited states in both the target and projectile as well as cross section of nucleon removal from the target. At energies of several hundred $\mathrm{MeV} /$ nucleon, the difference between $\sigma_{\mathrm{R}}$ and $\sigma_{\mathrm{I}}$ was found to be a few percent $[3,20,21]$ and was usually neglected to allow a direct comparison of the calculated $\sigma_{\mathrm{R}}$ with the measured $\sigma_{\mathrm{I}}$. Since the experimental uncertainty in the measured
$\sigma_{\mathrm{I}}$ is very small at the considered energies (around $1 \%$ for stable projectiles like ${ }^{4} \mathrm{He},{ }^{12} \mathrm{C}$, and $\left.{ }^{16} \mathrm{O}[1]\right)$ neglecting the difference between $\sigma_{\mathrm{R}}$ and $\sigma_{\mathrm{I}}$ might be too rough an approximation in comparing the calculated $\sigma_{\mathrm{R}}$ with the measured $\sigma_{\mathrm{I}}$ and testing nuclear radius at the accuracy level of $\pm 0.05 \mathrm{fm}$ or less $[1,22]$. In the present work, we try to estimate $\sigma_{\mathrm{I}}$ as accurately as possible by subtracting from the calculated $\sigma_{\mathrm{R}}$ the total cross section of the main inelastic scattering channels. Namely, we have calculated in DWIA, using the complex folded optical potential and inelastic form factors, the integrated cross sections $\sigma_{2^{+}}$and $\sigma_{3^{-}}$of inelastic scattering to the first excited $2^{+}$and $3^{-}$states of ${ }^{12} \mathrm{C}$ target at 4.44 and 9.64 $\mathrm{MeV}$, respectively. These states are known to have the largest cross sections in the inelastic proton and heavy ion scattering on ${ }^{12} \mathrm{C}$ at different energies. The deformation lengths used to construct transition densities (8) for the folding calculation were chosen so that the electric transition rates measured for these states are reproduced with the proton transition density as

$$
B(E \lambda \uparrow)=e^{2}\left|\int_{0}^{\infty} \rho_{\lambda}^{p}(r) r^{\lambda+2} d r\right|^{2} .
$$

Using a realistic Fermi distribution for the g.s. density of ${ }^{12} \mathrm{C}$ (see next Section) to generate the transition densities, we obtain $\delta_{2} \approx 1.54 \mathrm{fm}$ and $\delta_{3} \approx 2.11$ fm which reproduce the experimental transition rates $B(E 2 \uparrow) \approx 41 e^{2} \mathrm{fm}^{4}[23]$ and $B(E 3 \uparrow) \approx 750 e^{2} \mathrm{fm}^{6}$ [24], respectively, via Eq. (10). Since inelastic scattering to excited states of the unstable projectile is suppressed by a much faster breakup process, $\sigma_{\text {I }}$ can be approximately obtained as

$$
\begin{aligned}
\sigma_{\mathrm{I}} & =\sigma_{\mathrm{R}}-\sigma_{\text {Inel }} \\
& \approx \sigma_{\mathrm{R}}-\sigma_{2^{+}}-\sigma_{3^{-}}
\end{aligned}
$$

All the OM and DWIA calculations were made using the code ECIS97 [25] with the relativistic kinematics properly taken into account. At the energies around 1 $\mathrm{GeV} /$ nucleon the summation (1) is usually carried over up to 800 - 1000 partial waves to reach the full convergence of the $S$-matrix series for the considered nucleusnucleus systems.

\section{Adequacy and limitation of the folding approach}

Since the measured $\sigma_{\text {I }}$ have been analyzed extensively by different versions of Glauber model and its optical limit (OL) is sometimes referred to as the folding model $[6,26]$, we find it necessary to highlight the distinctive features of the present folding approach in comparison with the OL of Glauber model before going to discuss the results of calculation.

On the level of the nucleus-nucleus optical potential (OP), the present double-folding approach evaluates OP using fully finite-range $\mathrm{NN}$ interaction and taking into account the exchange effects accurately via the Fock term 
a)

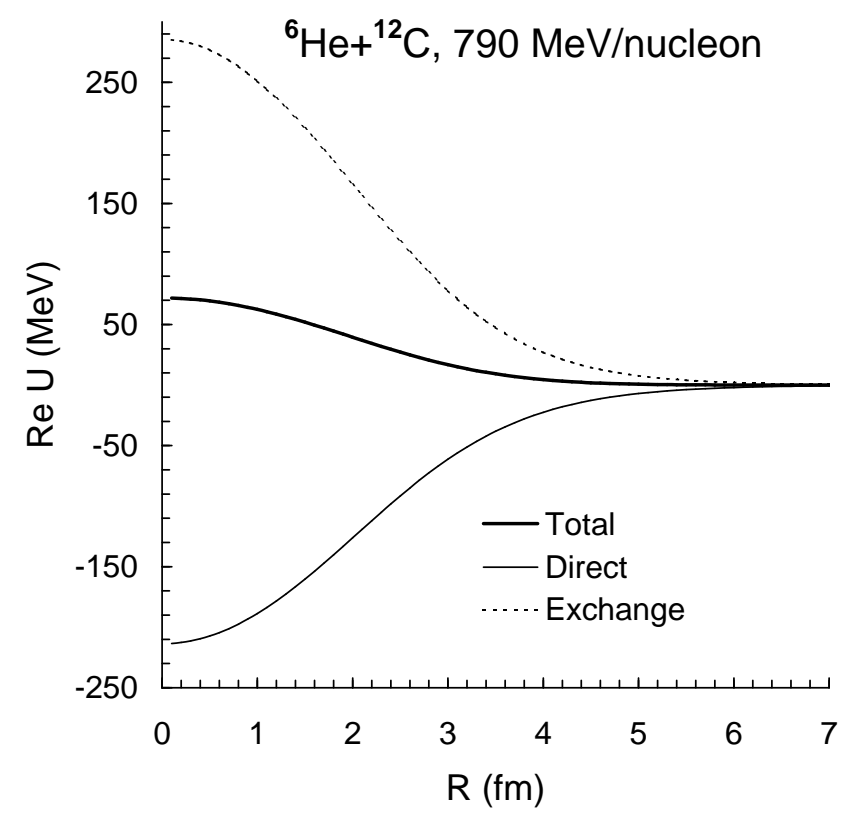

b)

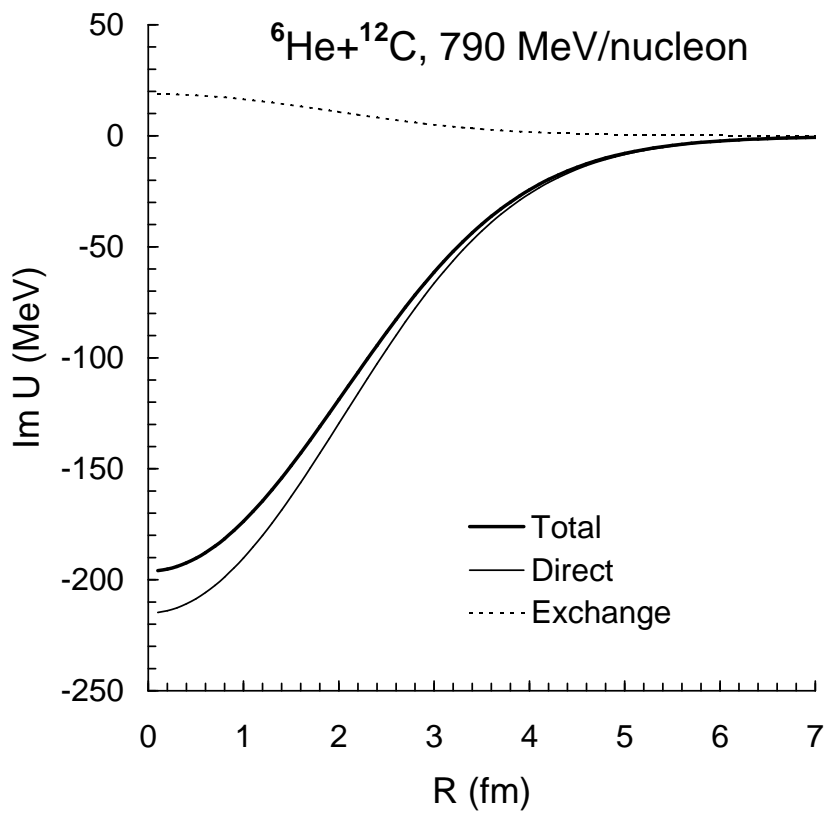

FIG. 1: Radial shape of the direct $V_{\mathrm{D}}$ and exchange $V_{\mathrm{Ex}}$ parts of the total optical potential $U$ for ${ }^{6} \mathrm{He}+{ }^{12} \mathrm{C}$ system at 790 $\mathrm{MeV} /$ nucleon. The real and imaginary parts of $U$ are shown in panels $a$ and $b$, respectively.

in Eq. (3). Therefore, individual nucleons are allowed to scatter after the collision into unoccupied single-particle states only. Sometimes, one discusses these effects as the exchange NN correlation. An appropriate treatment of the exchange $\mathrm{NN}$ correlation is indispensable not only in the folding calculation of OP and inelastic form factor, but also in the Hartree-Fock (HF) calculations of nuclear matter [27] and of the finite nuclei [28].

To obtain from the double-folding model presented above the simple expression of nucleus-nucleus OP used in the OL of Glauber model one needs to make a "doublezero" approximation which reduces the complex finiterange $t$-matrix interaction (9) to a zero-range (purely imaginary) NN scattering amplitude at zero NN angle $t_{\mathrm{NN}}\left(\theta=0^{\circ}\right) \delta(s)$ that can be further expressed through the total NN cross section $\sigma_{\mathrm{NN}}$, using the optical theorem. As a result, one needs to evaluate in the OL of Glauber model only a simple folding integral over local densities of the two colliding nuclei [6]

$$
U(\boldsymbol{R}) \rightarrow V_{O L}(\boldsymbol{R})=\frac{i \sigma_{\mathrm{NN}}}{2} \int \rho_{a}(\boldsymbol{R}) \rho_{A}\left(\boldsymbol{R}-\boldsymbol{r}_{A}\right) d^{3} r_{A}
$$

The prescription (12) is also known as the $t \rho \rho$ approximation [29] which neglects the off-shell part of the $t$ matrix. Besides the inaccuracy caused by the use of zerorange approximation [30], the zero-angle approximation takes into account only the on-shell $t$-matrix at zero momentum transfer (see Eq. (3) in Ref. [12]). Since the antisymmetrization of $t_{\mathrm{NN}}$ requires an accurate estimation of the NN knock-on exchange term which is strongest at large momentum transfers $\left(q>6 \mathrm{fm}^{-1}\right.$ at energies around $0.8 \mathrm{GeV}[12,13])$, the zero-angle approximation could strongly reduce the strength of the exchange term. A question remains, therefore, whether the NN antisymmetry is properly taken into account when one uses the empirical $\sigma_{\mathrm{NN}}$ in the Glauber folding integral (12). A similar aspect has been raised by Brandan et al. [31] who found that an overestimated absorption in the nucleusnucleus system (by the $t \rho \rho$ model) is due to the effects of Pauli principle. To illustrate the importance of the knock-on exchange term, we have plotted in Fig. 1 the direct and exchange components of the microscopic OP for ${ }^{6} \mathrm{He}+{ }^{12} \mathrm{C}$ system at $790 \mathrm{MeV} /$ nucleon predicted by our double-folding approach using realistic g.s. densities (see next Sect.) of the two colliding nuclei. One can see that the exchange term of the real OP is repulsive and much stronger than the (attractive) direct term, which makes the total real OP repulsive at all internuclear distances (see panel $a$ of Fig. 1). The exchange term of the imaginary OP is also repulsive but its relative strength is much weaker compared to that of the real OP, and the total imaginary OP remains attractive or absorptive at all distances. As a result, the direct part of the imaginary OP is about $10 \%$ more absorptive than the total imaginary OP (see panel $b$ of Fig. 1). The total reaction cross section predicted by the complex OP shown in Fig. 1 is $\sigma_{\mathrm{R}} \approx 727 \mathrm{mb}$. This value increases to $\sigma_{\mathrm{R}} \approx 750$ $\mathrm{mb}$ when the exchange potential $V_{\mathrm{EX}}$ is omitted in the $\mathrm{OM}$ calculation. Consequently, the relative contribution 
by the exchange term in $\sigma_{\mathrm{R}}$ is about $3 \%$. This difference is not small because it can lead to a difference of up to $7 \%$ in the extracted nuclear RMS radii. Due to an overwhelming contribution by the exchange part of the real $\mathrm{OP}$, the exchange potential affects the calculated elastic scattering cross section (see Fig. 2) much more substantially compared to $\sigma_{\mathrm{R}}$, which is determined mainly by the imaginary OP.

We will show below a slight (but rather systematic) difference in $\sigma_{\mathrm{R}}$ values obtained in our approach and the OL of Glauber model that might be due to the exchange effect. We note further that the elastic $S$-matrix is obtained in our approach rigorously from the quantal solution of the Schrödinger equation for elastic scattering wave, while the elastic $S$-matrix used in the Glauber model is given by the eikonal approximation which neglects the second-derivative term of the same Schrödinger equation.

A common feature of the present folding approach and the OL of Glauber model is the use of single-particle nuclear densities of the projectile and target as input for the calculation, leaving out all few-body correlations to the structure model used to construct the density. This simple ansatz has been referred to as "static density approximation" $[5,6]$ which does not take into account explicitly the dynamic few-body correlation between the core and valence nucleons in a loosely-bound projectile while it collides with the target. In the Glauber model, this type of few-body correlation can be treated explicitly $[3,5,6]$ using simple assumptions for the wave functions of the core and valence nucleons as well as that of their relative motion. For unstable nuclei with a well-extended halo structure, like ${ }^{11} \mathrm{Li}$ or ${ }^{6} \mathrm{He}$, such an explicit treatment of the dynamic few-body correlation leads consistently to a smaller $\sigma_{\mathrm{R}}$, i.e., to a larger nuclear radius compared to that given by the OL of Glauber model $[3,5,6]$. On the level of the HF-type folding calculation (3), an explicit treatment of the core and valence nucleons would result in a much more complicated triple-folding formalism which involves the antisymmetrization not only between the projectile nucleons and those of the target, but also between the nucleons of the core and the valence nucleons. Such an approach would clearly end up with a nonlocal OP which will not be easily used with the existing direct reaction codes. The lack of an appropriate treatment of the dynamic few-body correlations remains, therefore, the main limitation of the present folding approach in the calculation of the OP for systems involving unstable nuclei with halo-type structure.

Note that an effective way of taking into account the loose binding between the core and valence nucleons is to add a higher-order contribution from breakup (dynamic polarization potential) to the first-order folded potential $[21,32]$ or simply to renormalize the folded potential to fit the data. However, validity of the IA implies that higher-order multiple scattering or contribution from the dynamic polarization potential are negligible, and the folded OP and inelastic form factor based on the $t$-matrix interaction (9) should be used in the calculations without any further renormalization. Therefore, we will discuss below only results obtained with the unrenormalized folded potentials, keeping in mind possible effects due to the few-body correlation.

\section{RESULTS AND DISCUSSION}

\section{Results for stable $(N=Z)$ isotopes}

An important step in any experimental or theoretical reaction study with unstable beams is to gauge the method or model by the results obtained with stable beams. Therefore, we have considered first the available data of $\sigma_{\mathrm{I}}$ induced by stable ${ }^{4} \mathrm{He},{ }^{6} \mathrm{Li},{ }^{12} \mathrm{C}$, and ${ }^{16} \mathrm{O}$ beams on ${ }^{12} \mathrm{C}$ target [1]. These $(N=Z)$ nuclei are strongly bound, and the RMS radius of the (point) proton distribution inferred from the elastic electron scattering data [35] can be adopted as the "experimental" nuclear radius if the proton and neutron densities are assumed to be the same. To show the sensitivity of the calculated $\sigma_{\text {I }}$ to the nuclear radius, we present in Table I results obtained with different choices for the projectile density in each case. We use for the g.s. density of ${ }^{12} \mathrm{C}$ target a realistic Fermi (FM) distribution [16]

$$
\rho_{0}(r)=\rho_{0} /[1+\exp ((r-c) / a)]
$$

where $\rho_{0}=0.194 \mathrm{fm}^{-3}, c=2.214$ and $a=0.425 \mathrm{fm}$ were chosen to reproduce the shape of shell model density and experimental radius of $2.33 \mathrm{fm}$ for ${ }^{12} \mathrm{C}$.

${ }^{4} \mathrm{He}$ is a unique case where a simple harmonic oscillator $(\mathrm{HO})$ model can reproduce quite well its ground state density. If one chooses the $\mathrm{HO}$ parameter to give $\left.<r^{2}\right\rangle^{1 / 2}=1.461 \mathrm{fm}$ (close to the experimental radius of $1.47 \pm 0.02 \mathrm{fm}$ ) then one obtains the Gaussian form adopted in Ref. [7] for $\alpha$-density. This choice of ${ }^{4} \mathrm{He}$ density has been shown in the folding analysis of elastic $\alpha$-nucleus scattering [16] to be the most realistic. By comparing the calculated $\sigma_{\mathrm{I}}$ with the data, we find that this same choice of ${ }^{4} \mathrm{He}$ density gives the best agreement between $\sigma_{\mathrm{I}}^{\text {calc }}$ and $\sigma_{\mathrm{I}}^{\exp }$. Similar situation was found for ${ }^{12} \mathrm{C}$ and ${ }^{16} \mathrm{O}$ isotopes, where the best agreement with the data is given by the densities which reproduce the experimental nuclear radii. Beside a simple Fermi distribution [16], microscopic g.s. densities given by the Hartree-FockBogoliubov (HFB) calculation that takes into account the continuum [33] were also used. The agreement with the data for ${ }^{12} \mathrm{C}$ and ${ }^{16} \mathrm{O}$ given by the HFB densities is around $2 \%$, quite satisfactory for a fully microscopic structure model. We have further used $s p$-shell HO wave functions to construct the g.s. densities of ${ }^{6} \mathrm{Li},{ }^{12} \mathrm{C}$, and ${ }^{16} \mathrm{O}$. For ${ }^{12} \mathrm{C}$ and ${ }^{16} \mathrm{O}$, the best agreement with the $\sigma_{\mathrm{I}}$ data is again reached when the HO parameter is tuned to reproduce the experimental radii.

The agreement is slightly worse for ${ }^{6} \mathrm{Li}$ compared to ${ }^{4} \mathrm{He},{ }^{12} \mathrm{C}$, and ${ }^{16} \mathrm{O}$ cases if ${ }^{6} \mathrm{Li}$ density distribution reproduces the experimental radius. We have first used ${ }^{6} \mathrm{Li}$ 
TABLE I: The total reaction cross section $\sigma_{\mathrm{R}}$ and interaction cross section $\sigma_{\mathrm{I}}$ calculated for stable ${ }^{4} \mathrm{He},{ }^{6,7} \mathrm{Li},{ }^{12} \mathrm{C}$, and ${ }^{16} \mathrm{O}$ nuclei in comparison with $\sigma_{\mathrm{I}}^{\exp }$ taken from the data compilation in Ref. [1]. $\Delta \sigma_{\mathrm{I}}=\left|\sigma_{\mathrm{I}}^{\text {calc }}-\sigma_{\mathrm{I}}^{\exp }\right| / \sigma_{\mathrm{I}}^{\exp }$.

\begin{tabular}{|c|c|c|c|c|c|c|c|c|c|}
\hline Nucleus & $\begin{array}{c}\text { Energy } \\
(\mathrm{MeV} / \text { nucleon })\end{array}$ & Density Model & $\begin{array}{c}<r^{2}>^{1 / 2}{ }_{\text {calc }} \\
(\mathrm{fm})\end{array}$ & Reference & $\begin{array}{c}<r^{2}>\exp \\
(\mathrm{fm})\end{array}$ & $\begin{array}{l}\sigma_{\mathrm{R}}^{\text {calc }} \\
(\mathrm{mb})\end{array}$ & $\begin{array}{l}\sigma_{\mathrm{I}}^{\text {calc }} \\
(\mathrm{mb})\end{array}$ & $\begin{array}{l}\sigma_{\mathrm{I}}^{\exp } \\
(\mathrm{mb}) \\
\end{array}$ & $\begin{array}{l}\Delta \sigma_{\mathrm{I}} \\
(\%) \\
\end{array}$ \\
\hline${ }^{4} \mathrm{He}$ & 790 & $\begin{array}{l}\mathrm{HO} \\
\mathrm{HO} \\
\mathrm{HO}\end{array}$ & $\begin{array}{l}1.461 \\
1.550 \\
1.720\end{array}$ & $\begin{array}{c}7] \\
{[16]} \\
{[36]}\end{array}$ & $1.47 \pm 0.02^{a}$ & $\begin{array}{l}513 \\
523 \\
543\end{array}$ & $\begin{array}{l}504 \\
515 \\
536\end{array}$ & $503 \pm 5$ & $\begin{array}{l}0.2 \\
2.4 \\
6.6\end{array}$ \\
\hline${ }^{6} \mathrm{Li}$ & 790 & $\begin{array}{c}\text { IPM } \\
\mathrm{HO} \\
\mathrm{HO}\end{array}$ & $\begin{array}{l}2.401 \\
2.401 \\
2.320\end{array}$ & $\begin{array}{c}{[34]} \\
\text { This work } \\
\text { This work }\end{array}$ & $2.43 \pm 0.02^{a}$ & $\begin{array}{l}722 \\
723 \\
709 \\
\end{array}$ & $\begin{array}{l}717 \\
718 \\
703 \\
\end{array}$ & $688 \pm 10$ & $\begin{array}{l}4.2 \\
4.4 \\
2.2\end{array}$ \\
\hline${ }^{7} \mathrm{Li}$ & 790 & $\begin{array}{c}\text { IPM } \\
\mathrm{HO}\end{array}$ & $\begin{array}{l}2.367 \\
2.334\end{array}$ & $\begin{array}{c}{[34]} \\
\text { This work }\end{array}$ & $2.33 \pm 0.02^{b}$ & $\begin{array}{l}746 \\
744\end{array}$ & $\begin{array}{l}741 \\
739\end{array}$ & $736 \pm 6$ & $\begin{array}{l}0.7 \\
0.4\end{array}$ \\
\hline${ }^{12} \mathrm{C}$ & 950 & $\begin{array}{c}\mathrm{FM} \\
\mathrm{HO} \\
\mathrm{HFB}\end{array}$ & $\begin{array}{l}2.332 \\
2.332 \\
2.446\end{array}$ & $\begin{array}{c}16] \\
{[16]} \\
\text { This work }\end{array}$ & $2.33 \pm 0.02^{a}$ & $\begin{array}{l}854 \\
853 \\
881 \\
\end{array}$ & $\begin{array}{l}844 \\
843 \\
872 \\
\end{array}$ & $853 \pm 6$ & $\begin{array}{l}1.1 \\
1.1 \\
2.2\end{array}$ \\
\hline${ }^{16} \mathrm{O}$ & 970 & $\begin{array}{c}\mathrm{FM} \\
\mathrm{HO} \\
\mathrm{HFB}\end{array}$ & $\begin{array}{l}2.618 \\
2.612 \\
2.674\end{array}$ & $\begin{array}{c}{[16]} \\
{[16]} \\
\text { This work }\end{array}$ & $2.61 \pm 0.01^{a}$ & $\begin{array}{c}992 \\
988 \\
1006\end{array}$ & $\begin{array}{l}981 \\
978 \\
997\end{array}$ & $982 \pm 6$ & $\begin{array}{l}0.1 \\
0.4 \\
1.4\end{array}$ \\
\hline
\end{tabular}

${ }^{a}$ RMS radius of the proton density given by the experimental charge density [35] unfolded with the finite size of proton.

${ }^{b}$ Nuclear RMS radius deduced from the Glauber model analysis of the same $\sigma_{\mathrm{I}}$ data in the OL approximation [1].

density given by the independent particle model (IPM) developed by Satchler [7, 34] which generates realistic wave function for each single-particle orbital using a Woods-Saxon (WS) potential for the bound state problem. The IPM density gives $\left\langle r^{2}>^{1 / 2} \approx 2.40 \mathrm{fm}\right.$ for ${ }^{6} \mathrm{Li}$, rather close to the experimental radius of $2.43 \pm 0.02$ fm inferred from $(e, e)$ data [35]. The HO density gives the same $\sigma_{\mathrm{I}}$ as that given by the IPM density if the HO parameter is chosen to give the same radius of $2.40 \mathrm{fm}$. These two versions of ${ }^{6} \mathrm{Li}$ density overestimate the $\sigma_{\mathrm{I}}$ data by about $4 \%$. If the $\mathrm{HO}$ parameter is chosen to give $\left\langle r^{2}\right\rangle^{1 / 2} \approx 2.32 \mathrm{fm}$ then the agreement with the $\sigma_{\mathrm{I}}$ data improves to around $2 \%$. This result indicates that our folding + DWIA analysis slightly overestimates the absorption in ${ }^{6} \mathrm{Li}+{ }^{12} \mathrm{C}$ system. Since ${ }^{6} \mathrm{Li}$ is a loosely bound $\alpha+d$ system, this few percent discrepancy with the $\sigma_{\text {I }}$ data might well be due to the dynamic correlation between the $\alpha$-core and deuteron cluster in ${ }^{6} \mathrm{Li}$ during the collision which is not taken into account by our approach. Note that a few-body Glauber calculation [6] (which takes into account explicitly the dynamic correlation between $\alpha$ and $d$ ) ends up, however, with about the same discrepancy (see Fig. 4 in Ref. [6]). ${ }^{6} \mathrm{Li}$ remains, therefore, an interesting case for the reaction models to improve their ingredients. For ${ }^{7} \mathrm{Li}$, the IPM density [7] gives $<r^{2}>_{p}^{1 / 2} \approx 2.28 \mathrm{fm}$ (close to the experimental value of $2.27 \pm 0.01 \mathrm{fm}$ [35]) and $\left\langle r^{2}>_{n}^{1 / 2} \approx 2.43 \mathrm{fm}\right.$ which make the matter radius $\left\langle r^{2}>^{1 / 2} \approx 2.37 \mathrm{fm}\right.$. As a result, $\sigma_{\text {I }}$ calculated with the IPM density for ${ }^{7} \mathrm{Li}$ agrees with the data within less than $1 \%$. In the $\mathrm{HO}$ model for ${ }^{7} \mathrm{Li}$ density, we have chosen the HO parameter for protons to reproduce the experimental radius of $2.27 \mathrm{fm}$ and that for neutrons adjusted by the best agreement with the $\sigma_{\mathrm{I}}$ data. The best-fit $<r^{2}>^{1 / 2}$ radius then becomes around $2.33 \mathrm{fm}$.
We conclude from these results that the present folding + DWIA approach and local $t$-matrix interaction by Franey and Love [13] are quite suitable for the description of the nucleus-nucleus interaction cross section at energies around $1 \mathrm{GeV} /$ nucleon, with the prediction accuracy as fine as 1-2\% for the stable and strongly bound nuclei.

\section{Results for neutron-rich isotopes}

Our results for neutron-rich $\mathrm{He}, \mathrm{Li}, \mathrm{C}$, and $\mathrm{O}$ isotopes are presented in Table II. Since ${ }^{6} \mathrm{He}$ beams are now available with quite a good resolution, this nucleus is among the most studied unstable nuclei. In the present work we have tested 3 different choices for ${ }^{6} \mathrm{He}$ density in the calculation of $\sigma_{\mathrm{I}}$. The microscopic ${ }^{6} \mathrm{He}$ density obtained in a HF calculation [30] has a rather small radius $\left\langle r^{2}\right\rangle^{1 / 2} \approx 2.20 \mathrm{fm}$ and the calculated $\sigma_{\mathrm{I}}$ underestimates the data by about $5 \%$. A larger radius of $2.53 \mathrm{fm}$ is given by the density obtained in a consistent three-body formalism [5] and the corresponding $\sigma_{\mathrm{I}}$ agrees better with the data. Given an accurate ${ }^{7} \mathrm{Li}$ density obtained in the IPM [7] as shown above and the fact that ${ }^{6} \mathrm{He}$ can be produced by a proton-pickup reaction on ${ }^{7} \mathrm{Li}$, we have constructed the g.s. density of ${ }^{6} \mathrm{He}$ in the IPM (with the recoil effect properly taken into account [34]) using the following WS parameters for the single-particle states: $r_{0}=1.25 \mathrm{fm}, a=0.65 \mathrm{fm}$ for the $s \frac{1}{2}$ neutrons and protons which are bound by $S_{n}=25 \mathrm{MeV}$ and $S_{p}=23$ $\mathrm{MeV}$, respectively; $r_{0}=1.35 \mathrm{fm}, a=0.65 \mathrm{fm}$ for the $p \frac{3}{2}$ halo neutrons which are bound by $S_{n}=1.86 \mathrm{MeV}$. The WS depth is adjusted in each case to reproduce the binding energy. The obtained IPM density gives the proton, neutron and total nuclear radii of ${ }^{6} \mathrm{He}$ as $1.755,2.746$ and $2.460 \mathrm{fm}$, respectively. This choice of ${ }^{6} \mathrm{He}$ density 
TABLE II: The same as Table I but for neutron-rich He, Li, C, and O isotopes. Note that $<r^{2}>_{\text {calc }}^{1 / 2}$ given by the HO densities should have about the same uncertainties as those deduced for $\left\langle r^{2}>_{\exp }^{1 / 2}\right.$ by the OL of Glauber model.

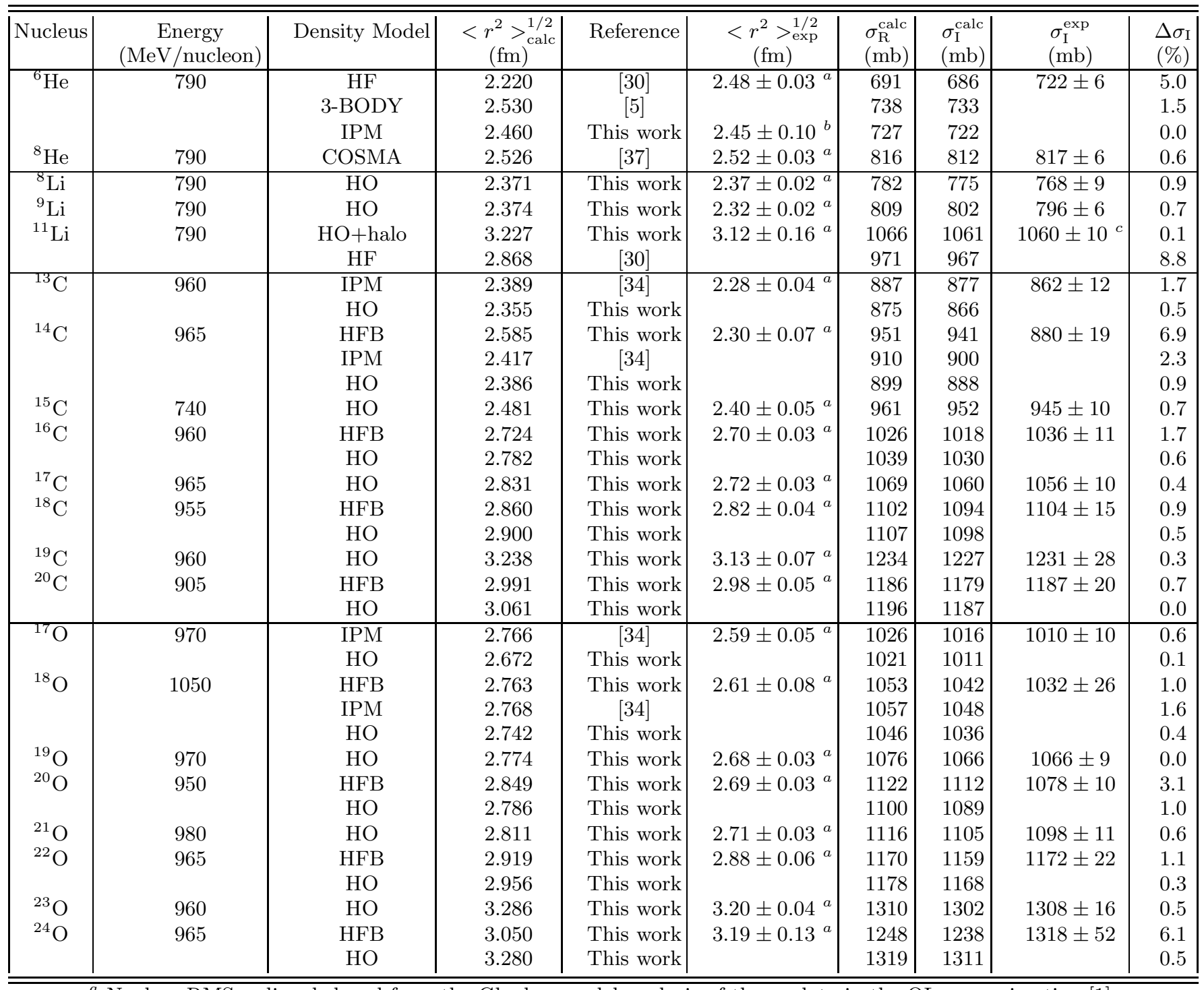

${ }^{a}$ Nuclear RMS radius deduced from the Glauber model analysis of the $\sigma_{\mathrm{I}}$ data in the OL approximation [1].

${ }^{b}$ Nuclear RMS radius deduced from the Glauber model analysis of elastic ${ }^{6} \mathrm{He}+\mathrm{p}$ scattering data at $0.7 \mathrm{GeV} / \mathrm{nucleon}[38]$. ${ }^{c} \sigma_{\text {I }}$ data taken from Ref. [43].

also gives the best agreement with the $\sigma_{\mathrm{I}}$ data. We note that a Glauber model analysis of the elastic ${ }^{6} \mathrm{He}+\mathrm{p}$ scattering at $0.7 \mathrm{GeV} /$ nucleon [38], which takes into account higher-order multiple scattering effects, gives a best-fit $\left\langle r^{2}\right\rangle^{1 / 2} \approx 2.45 \mathrm{fm}$ for ${ }^{6} \mathrm{He}$, very close to our result. Since elastic ${ }^{6} \mathrm{He}+{ }^{12} \mathrm{C}$ scattering has recently been measured at lower energies [40], we found it interesting to plot the 3 densities and elastic ${ }^{6} \mathrm{He}+{ }^{12} \mathrm{C}$ scattering cross sections at $790 \mathrm{MeV} /$ nucleon predicted by the corresponding complex folded OP (the radial shape of the OP obtained with the IPM density for ${ }^{6} \mathrm{He}$ is shown in Fig. 1). As can be seen from Fig. 2, the IPM density has the neutronhalo tail very close to that of the density calculated in the three-body model [5] and they both give a good descrip- tion of $\sigma_{\mathrm{I}}$. The predicted elastic cross section is strongly forward peaked and the difference in densities begins to show up after the first diffractive maximum. Such a measurement should be feasible at the facilities used for elastic ${ }^{6} \mathrm{He}+\mathrm{p}$ scattering at $0.7 \mathrm{GeV} /$ nucleon [38] and would be very helpful in testing finer details of ${ }^{6} \mathrm{He}$ density. As already discussed in previous Sect., the exchange part of the microscopic OP affects the elastic cross section very strongly (see dotted curve in panel $b$ of Fig. 2) and the elastic ${ }^{6} \mathrm{He}+{ }^{12} \mathrm{C}$ scattering measurement would be also a very suitable probe of the exchange effects in this system.

Since ${ }^{6} \mathrm{He}$ is a loosely bound halo nucleus with a well established three-body $\alpha+n+n$ structure, the dynamic correlation between the $\alpha$-core and dineutron is expected to 
a)

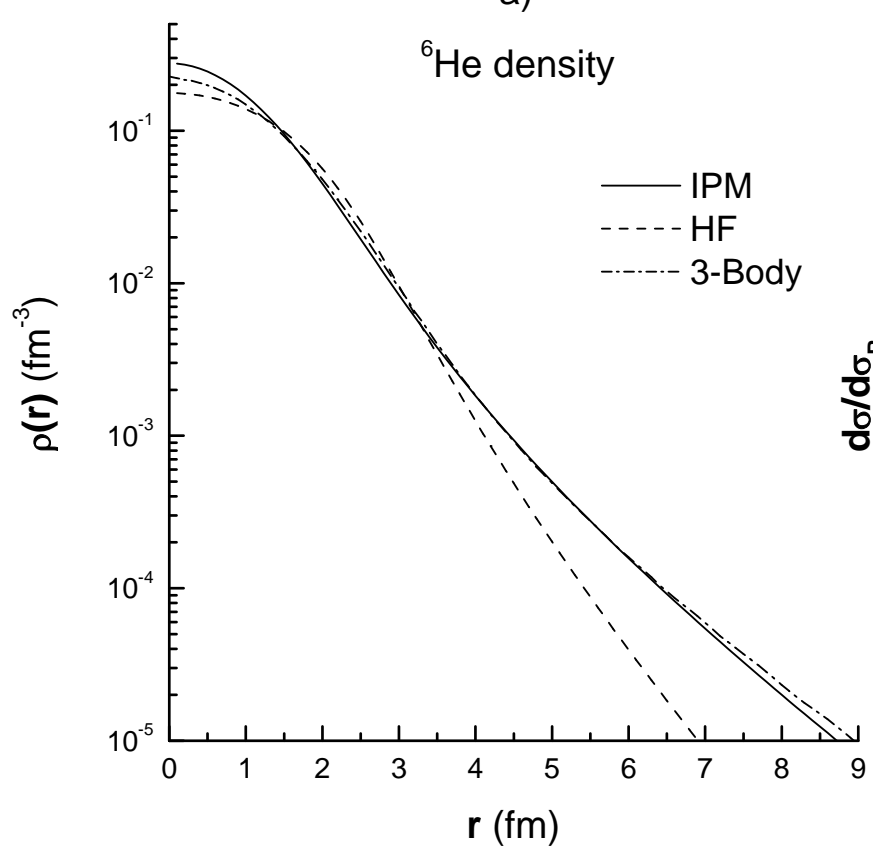

b)

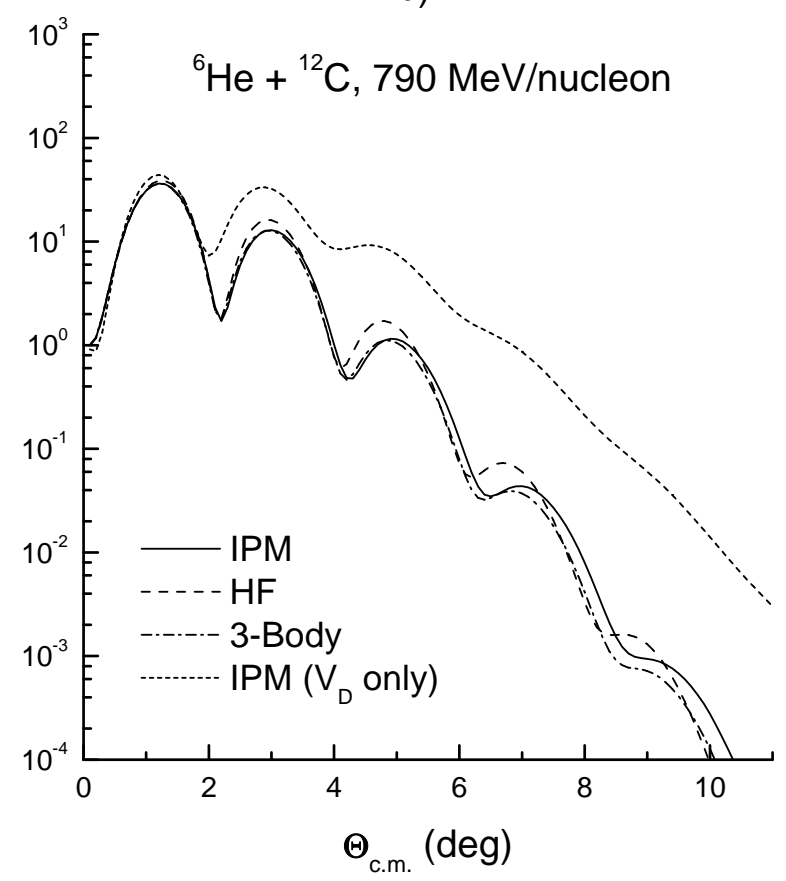

FIG. 2: Three versions of ${ }^{6} \mathrm{He}$ g.s. density used in the folding calculation (panel $a$ ) and elastic ${ }^{6} \mathrm{He}+{ }^{12} \mathrm{C}$ scattering cross sections at $790 \mathrm{MeV} /$ nucleon obtained with the corresponding complex folded optical potentials (panel $b$ ). The dotted curve in panel $b$ is obtained without the exchange part of the OP.

be important during the collision. Our folding + DWIA approach using three-body density for ${ }^{6} \mathrm{He}$ (version FC [5]) gives $\sigma_{\mathrm{I}} \approx 733 \mathrm{mb}$ compared to about $720 \mathrm{mb}$ given by the few-body calculation by Tostevin et al. (see Fig. 4 in Ref. [6]) based on the same three-body wave function for ${ }^{6} \mathrm{He}$. The difference in the calculated $\sigma_{\mathrm{I}}$ leads to an increase of about $2-3 \%$ in the $<r^{2}>^{1 / 2}$ value. It is likely that such a difference is, in part, due to the dynamic correlation between the $\alpha$-core and dineutron which was not considered in our folding + DWIA approach. For ${ }^{8} \mathrm{He}$ nucleus, the OL of Glauber analysis of $\sigma_{\text {I }}$ data [1] and the multiple-scattering Glauber analysis of elastic ${ }^{8} \mathrm{He}+\mathrm{p}$ data at $0.7 \mathrm{GeV} /$ nucleon [38] give $\left\langle r^{2}\right\rangle^{1 / 2}$ around 2.52 and $2.53 \mathrm{fm}$, respectively. By using the microscopic ${ }^{8} \mathrm{He}$ density obtained in a four-body (COSMA) model [37], which gives $\left\langle r^{2}\right\rangle^{1 / 2}=2.526 \mathrm{fm}$, our folding + DWIA approach reproduces the measured $\sigma_{\text {I }}$ data within less than $1 \%$. Note that a (multiple scattering) Glauber model analysis of the elastic ${ }^{6,8} \mathrm{He}+\mathrm{p}$ scattering at $0.7 \mathrm{GeV} /$ nucleon which takes into account the dynamic few-body correlation explicitly was done by Al-Khalili and Tostevin [39], and they have obtained the best-fit nuclear radii of about 2.5 and $2.6 \mathrm{fm}$ for ${ }^{6} \mathrm{He}$ and ${ }^{8} \mathrm{He}$, respectively, around $2 \%$ larger than our results.

\section{Parameters of $\mathrm{HO}$ densities deduced from $\sigma_{\mathrm{I}}$ data}

Although the HO model is a very simple approach, the HO densities were shown above to be useful in testing the nuclear radii for stable $(N=Z)$ nuclei. Moreover, the HO-type densities (with the appropriately chosen HO lengths) for the $s d$-shell nuclei have been successfully used in the analysis of (e,e) data, measurements of isotope shift and muonic atoms [1]. Therefore, it is not unreasonable to use simple $\mathrm{HO}$ parameterization for the g.s. densities of neutron-rich nuclei to estimate the nuclear radii, based on our folding + DWIA analysis of $\sigma_{\text {I }}$ data. For a $N \neq Z$ nucleus, one needs to generate proton and neutron densities separately as

$$
\rho_{\tau}(r)=\frac{2}{\pi^{3 / 2} b_{\tau}^{3}}\left(1+P_{\tau} \frac{r^{2}}{b_{\tau}^{2}}+D_{\tau} \frac{r^{4}}{b_{\tau}^{4}}\right) \exp \left(-\frac{r^{2}}{b_{\tau}^{2}}\right)
$$

where $\tau=n$ or $p$, parameters $P_{\tau}$ and $D_{\tau}$ are determined from the nucleon occupation of the $p$ - and $d$ harmonicoscillator shells, respectively.

To generate the g.s. densities of ${ }^{8,9} \mathrm{Li}$ isotopes, we have assumed the proton density of these nuclei to be approximately that of ${ }^{7} \mathrm{Li}$ and the neutron $\mathrm{HO}$ length $b_{n}$ is adjusted in each case to reproduce the measured $\sigma_{\mathrm{I}}$ (see Tables II and III). While the obtained $<r^{2}>^{1 / 2}$ for ${ }^{8} \mathrm{Li}$ is rather close to that given by the OL of Glauber model [1], results obtained for ${ }^{9} \mathrm{Li}$ are different and we could reproduce the $\sigma_{\mathrm{I}}$ data only if the neutron $\mathrm{HO}$ length is 
TABLE III: The HO-density parameters (14) for neutron-rich Li, C, and O isotopes.

\begin{tabular}{|c|c|c|c|c|c|c|c|c|c|}
\hline Nucleus & $P_{n}$ & $P_{p}$ & $D_{n}$ & $D_{p}$ & $\begin{array}{c}b_{n} \\
(\mathrm{fm})\end{array}$ & $\begin{array}{c}b_{p} \\
(\mathrm{fm})\end{array}$ & $\begin{array}{c}<r^{2}>_{n}^{1 / 2} \\
(\mathrm{fm})\end{array}$ & $\begin{array}{c}<r^{2}>_{p}^{1 / 2} \\
(\mathrm{fm})\end{array}$ & $\begin{array}{c}<r^{2}>^{1 / 2} \\
(\mathrm{fm})\end{array}$ \\
\hline${ }^{7} \mathrm{Li}$ & $2 / 3$ & $1 / 3$ & 0.0 & 0.0 & 1.684 & 1.6766 & 2.382 & 2.270 & 2.334 \\
\hline${ }^{8} \mathrm{Li}$ & 1.0 & $1 / 3$ & 0.0 & 0.0 & 1.6770 & 1.6776 & 2.430 & 2.270 & 2.371 \\
\hline${ }^{9} \mathrm{Li}$ & $4 / 3$ & $1 / 3$ & 0.0 & 0.0 & 1.6470 & 1.6766 & 2.424 & 2.270 & 2.374 \\
\hline${ }^{13} \mathrm{C}$ & $5 / 3$ & $4 / 3$ & 0.0 & $\overline{0.0}$ & 1.6058 & 1.5722 & 2.389 & 2.314 & 2.355 \\
\hline${ }^{14} \mathrm{C}$ & 2.0 & $4 / 3$ & 0.0 & 0.0 & 1.6226 & 1.5762 & 2.434 & 2.320 & 2.386 \\
\hline${ }^{15} \mathrm{C}$ & 2.0 & $4 / 3$ & $2 / 15$ & 0.0 & 1.6630 & 1.5898 & 2.570 & 2.340 & 2.481 \\
\hline${ }^{16} \mathrm{C}$ & 2.0 & $4 / 3$ & $4 / 15$ & 0.0 & 1.8512 & 1.7128 & 2.927 & 2.521 & 2.782 \\
\hline${ }^{17} \mathrm{C}$ & 2.0 & $4 / 3$ & $2 / 5$ & 0.0 & 1.8552 & 1.7128 & 2.986 & 2.521 & 2.831 \\
\hline${ }^{18} \mathrm{C}$ & 2.0 & $4 / 3$ & $8 / 15$ & 0.0 & 1.8752 & 1.7297 & 3.062 & 2.546 & 2.900 \\
\hline${ }^{19} \mathrm{C}$ & 2.0 & $4 / 3$ & $2 / 3$ & 0.0 & 2.1252 & 1.7297 & 3.512 & 2.546 & 3.238 \\
\hline${ }^{20} \mathrm{C}$ & 2.0 & $4 / 3$ & $4 / 5$ & 0.0 & 1.9462 & 1.7467 & 3.248 & 2.571 & 3.061 \\
\hline${ }^{17} \mathrm{O}$ & 2.0 & 2.0 & $2 / 15$ & 0.0 & 1.7775 & 1.7232 & 2.747 & 2.585 & 2.672 \\
\hline${ }^{18} \mathrm{O}$ & 2.0 & 2.0 & $4 / 15$ & 0.0 & 1.7601 & 1.7935 & 2.783 & 2.690 & 2.742 \\
\hline${ }^{19} \mathrm{O}$ & 2.0 & 2.0 & $2 / 5$ & 0.0 & 1.7601 & 1.7935 & 2.833 & 2.690 & 2.774 \\
\hline${ }^{20} \mathrm{O}$ & 2.0 & 2.0 & $8 / 15$ & 0.0 & 1.7401 & 1.8005 & 2.842 & 2.701 & 2.786 \\
\hline${ }^{21} \mathrm{O}$ & 2.0 & 2.0 & $2 / 3$ & 0.0 & 1.7401 & 1.8005 & 2.876 & 2.701 & 2.811 \\
\hline${ }^{22} \mathrm{O}$ & 2.0 & 2.0 & $4 / 5$ & 0.0 & 1.8498 & 1.8081 & 3.087 & 2.712 & 2.956 \\
\hline${ }^{23} \mathrm{O}$ & 2.0 & 2.0 & $14 / 15$ & 0.0 & 2.1118 & 1.8081 & 3.555 & 2.712 & 3.286 \\
\hline${ }^{24} \mathrm{O}$ & 2.0 & 2.0 & $16 / 15$ & 0.0 & 2.0758 & 1.8261 & 3.520 & 2.739 & 3.280 \\
\hline
\end{tabular}

chosen to give $\left\langle r^{2}>_{\text {calc }}^{1 / 2} \approx 2.37\right.$ fm or about $2 \%$ larger than that given by the OL of Glauber model. For the halo nucleus ${ }^{11} \mathrm{Li}$, a ${ }^{9} \mathrm{Li}$ core + two-neutron halo model was used to generate its density. Namely, we have used $\mathrm{HO}$ density of ${ }^{9} \mathrm{Li}$ that reproduces the measured $\sigma_{\mathrm{I}}$ for ${ }^{9} \mathrm{Li}$ and a Gaussian tail for the two-neutron halo density. To reach a best agreement between $\sigma_{\mathrm{I}}^{\text {exp }}$ taken from Ref. [43] and $\sigma_{\mathrm{I}}^{\text {calc }}$, the Gaussian range was chosen to give $<r^{2}>_{\text {calc }}^{1 / 2} \approx 3.23 \mathrm{fm}$ which is about $0.1 \mathrm{fm}$ larger than that given by the OL of Glauber model [1]. A microscopic density for ${ }^{11} \mathrm{Li}$ obtained in the $\mathrm{HF}$ calculation [30] (which gives $<r^{2}>^{1 / 2}=2.868 \mathrm{fm}$ ) has also been used in our folding analysis. The agreement with the data becomes much worse in this case (see Table II) and we conclude that the radius given by the HF density is somewhat too small. To show the sensitivity of our analysis to the nuclear radius, we have plotted in Fig. $3 \sigma_{\text {I }}$ predicted by three versions of ${ }^{11} \mathrm{Li}$ density with the Gaussian range of the $2 n$-halo adjusted to give $<r^{2}>^{1 / 2}=3.15,3.23$ and $3.30 \mathrm{fm}$, respectively, compared to $\sigma_{\mathrm{I}}^{\exp }=1060 \pm 10$ $\mathrm{mb}$ [43]. It is easily to infer from Fig. 3 an empirical RMS radius of $3.23 \pm 0.05 \mathrm{fm}$ for ${ }^{11} \mathrm{Li}$. Note that $\sigma_{\mathrm{I}}$ measurement for ${ }^{11} \mathrm{Li}+{ }^{12} \mathrm{C}$ system at $790 \mathrm{MeV} /$ nucleon has been reported in several works with $\sigma_{\mathrm{I}}^{\exp }=1040 \pm 60$ [42], $1047 \pm 40$ [41] and $1060 \pm 10 \mathrm{mb}$ [43]. If we adjust Gaussian range of the $2 n$-halo in ${ }^{11} \mathrm{Li}$ density to reproduce these $\sigma_{\mathrm{I}}^{\exp }$ values, the corresponding $\left\langle r^{2}>^{1 / 2}\right.$ radii of ${ }^{11} \mathrm{Li}$ are $3.13,3.15$ and $3.23 \mathrm{fm}$, respectively. Since $\sigma_{\text {I }}$ data obtained in Ref. [43] has a much better statistics and less uncertainty, we have adopted $<r^{2}>^{1 / 2}=$ $3.23 \pm 0.05 \mathrm{fm}$ as the most realistic RMS radius of ${ }^{11} \mathrm{Li}$ given by our folding + DWIA analysis.

The total reaction cross section for ${ }^{11} \mathrm{Li}+{ }^{12} \mathrm{C}$ system at $790 \mathrm{MeV} /$ nucleon has been studied earlier in the fewbody Glauber formalism by Al-Khalili et al. [5], where $<r^{2}>^{1 / 2}$ radius for ${ }^{11} \mathrm{Li}$ was shown to increase from $3.05 \mathrm{fm}$ (in the OL) to around $3.53 \mathrm{fm}$ when the dynamic correlation between ${ }^{9} \mathrm{Li}$-core and $2 n$-halo during the collision is treated explicitly. This is about $9 \%$ larger than $\left.<r^{2}\right\rangle^{1 / 2}$ radius obtained in our folding + DWIA approach based on the same $\sigma_{\text {I }}$ data. Although various structure calculations for ${ }^{11} \mathrm{Li}$ give its RMS radius around 3.1-3.2 fm (see Refs. [1, 4] and references therein), a very recent coupled-channel three-body model for ${ }^{11} \mathrm{Li}$ by Ikeda et al. [44, 45] shows that its RMS radius is ranging from 3.33 to $3.85 \mathrm{fm}$ if the $2 n$-halo wave function consists of 21 to $39 \%$ mixture from $\left(s \frac{1}{2}\right)^{2}$ state, respectively. A comparison of the calculated Coulomb breakup cross section with the data [45] suggests that this $s$-wave mixture is around 20-30\%. Thus, the nuclear radius of ${ }^{11} \mathrm{Li}$ must be larger than that accepted sofar $[1,4]$ and be around $3.3-3.5 \mathrm{fm}$, closer to the result of the few-body calculation [5] and the upper limit of RMS radius given by our folding + DWIA analysis.

For most of neutron-rich $\mathrm{C}$ and $\mathrm{O}$ isotopes considered here, we have first fixed the proton $\mathrm{HO}$ lengths $b_{p}$ to reproduce the proton $<r^{2}>_{p}^{1 / 2}$ radii predicted by the microscopic IPM and HFB densities (as described below). The neutron HO lengths $b_{n}$ are then adjusted to the best agreement with $\sigma_{\text {I }}$ data, and the obtained HO parameters are summarized in Table III. 


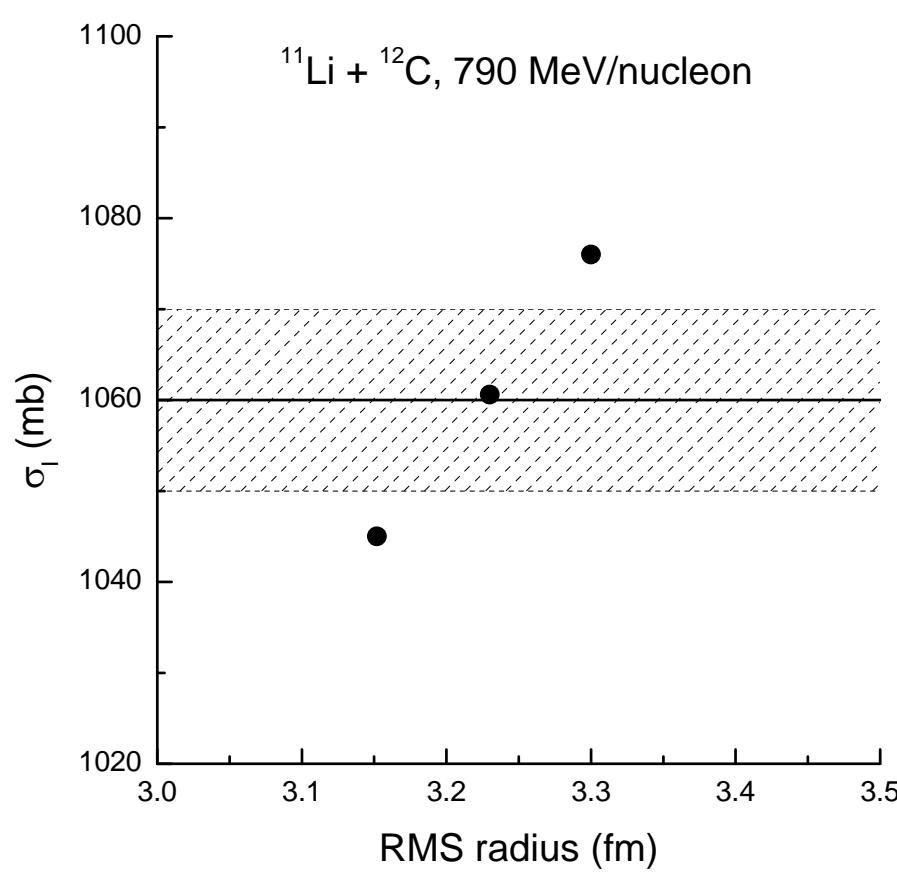

FIG. 3: $\sigma_{\mathrm{I}}^{\text {calc }}$ obtained with three versions of ${ }^{11} \mathrm{Li}$ g.s. density, where Gaussian range of the $2 n$-halo was adjusted to give $<r^{2}>^{1 / 2}=3.15,3.23$ and $3.30 \mathrm{fm}$ for ${ }^{11} \mathrm{Li}$, in comparison with $\sigma_{\mathrm{I}}^{\exp }=1060 \pm 10 \mathrm{mb}$ [43].

\section{Microscopic HFB densities}

Before discussing the results obtained for the neutronrich $\mathrm{C}$ and $\mathrm{O}$ isotopes, we give here a brief description of the microscopic HFB approach used to calculate the g.s. densities of even $\mathrm{C}$ and $\mathrm{O}$ isotopes. More details about this approach can be found in Ref. [33].

We solve the HFB equations in coordinate representation and in spherical symmetry with the inclusion of continuum states for neutron-rich nuclei. As the neutron Fermi energies of these nuclei are typically quite close to zero, pairing correlations can easily scatter pairs of neutrons from the bound states towards continuum states. For this reason, the inclusion and the treatment of continuum states in the calculation are very important. In our calculation the continuum is treated exactly, i.e., with the correct boundary conditions for continuum wave functions and by taking into account the widths of the resonances. Resonant states are localized by studying the behavior of the phase shifts with respect to the quasi-particle energy for each partial wave $(l, j)$.

The calculations were done with the Skyrme interaction SLy4 for the mean field channel and with the follow- ing zero-range density-dependent interaction

$$
V=V_{0}\left[1-\left(\frac{\rho(r)}{\rho_{0}}\right)^{\gamma}\right] \delta\left(\boldsymbol{r}_{1}-\boldsymbol{r}_{2}\right)
$$

for the pairing channel. In Eq. (15), $\rho_{0}$ is the saturation density and $\gamma$ is chosen equal to 1 . We have adapted the prescription of Refs. [46, 47] to finite nuclei in order to fix $V_{0}$ together with the quasi-particle energy cutoff. This prescription, requiring that the free neutronneutron scattering length has to be reproduced in the truncated space, allows us to deduce a relation between the parameter $V_{0}$ and the quasiparticle energy cutoff.

\section{Nuclear radii of Carbon and Oxygen isotopes}

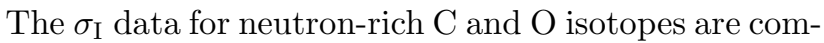
pared in Table II with $\sigma_{\text {I }}$ predicted by different choices of nuclear densities. We have tested first the IPM density for ${ }^{13} \mathrm{C}$ [34] based on the single-particle spectroscopic factors obtained in the shell model by Cohen and Kurath [48]. This IPM density gives $\left\langle r^{2}>^{1 / 2} \approx 2.39 \mathrm{fm}\right.$ for ${ }^{13} \mathrm{C}$ and the predicted $\sigma_{\mathrm{I}}$ agrees with the data within less than $2 \%$. We have further made IPM calculation for ${ }^{14} \mathrm{C}$ based on the same single-particle configurations, with the WS parameters for $s p$-shells appropriately corrected for the recoil effects and experimental nucleon separation energies $S_{n, p}$ of ${ }^{14} \mathrm{C}$. This IPM density gives $<r^{2}>^{1 / 2} \approx 2.42$ fm for ${ }^{14} \mathrm{C}$ and the predicted $\sigma_{\mathrm{I}}$ also agrees with the data within $2 \%$. The $\mathrm{HO}$ densities were also parameterized for ${ }^{13,14} \mathrm{C}$ with the proton $\mathrm{HO}$ lengths $b_{p}$ chosen to reproduce $\left\langle r^{2}>_{p}^{1 / 2}\right.$ values predicted by the IPM. The best-fit neutron $\mathrm{HO}$ lengths $b_{n}$ result in $\left\langle r^{2}\right\rangle^{1 / 2}=2.36$ and $2.39 \mathrm{fm}$ for ${ }^{13} \mathrm{C}$ and ${ }^{14} \mathrm{C}$, respectively. These values agree fairly with those given by the IPM densities. The microscopic $\mathrm{HFB}$ density gives for ${ }^{14} \mathrm{C}$ a significantly larger $<r^{2}>^{1 / 2}$ radius of $2.59 \mathrm{fm}$ and the calculated $\sigma_{\mathrm{I}}$ overestimates the data by nearly $7 \%$. Note that the OL of Glauber model gives smaller radius of 2.28 and $2.30 \mathrm{fm}$ for ${ }^{13} \mathrm{C}$ and ${ }^{14} \mathrm{C}$, respectively, based on the same $\sigma_{\mathrm{I}}$ data [1]. This means that the absorption given by the OL of Glauber model is indeed stronger than that given by our approach, as expected from discussion in Sect. II.

For the neutron-rich even ${ }^{16-20} \mathrm{C}$ isotopes, the $\mathrm{HFB}$ densities give a remarkably better agreement with the data and it is, therefore, reasonable to fix the proton $\mathrm{HO}$ lengths of the $\mathrm{HO}$ densities for each of ${ }^{15-20} \mathrm{C}$ isotopes to reproduce $<r^{2}>_{p}^{1 / 2}$ radius predicted by the HFB calculation for the nearest even neighbor. The best-fit neutron $\mathrm{HO}$ lengths result in the nuclear radii quite close to those given by the HFB densities (see Tables II and III). We emphasize that the nuclear radii given by our analysis, using the $\mathrm{HO}$ densities for $\mathrm{C}$ isotopes, are about $0.1 \mathrm{fm}$ larger than those deduced from the OL of Glauber model [1]. Given a high sensitivity of $\sigma_{\text {I }}$ data to the nuclear size, a difference of $0.1 \mathrm{fm}$ is not negligible.

To illustrate the mass dependence of the nuclear radius, we have plotted in Fig. 4a the RMS radii given 

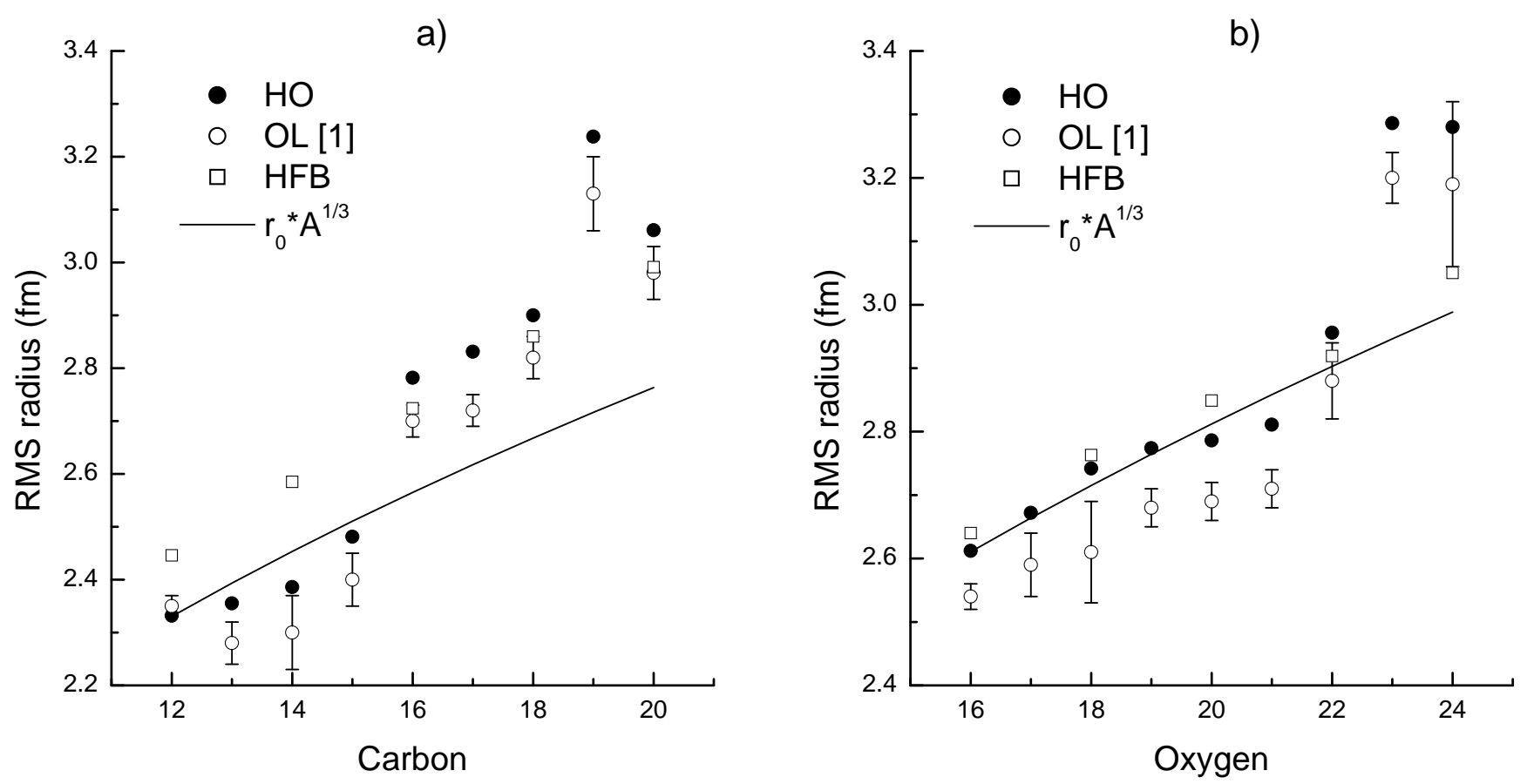

FIG. 4: Mass dependence of the nuclear RMS radius for Carbon (panel $a$ ) and Oxygen (panel $b$ ) isotopes given by the two choices (HFB and HO) of the g.s. densities compared to that deduced from the Glauber model analysis in the OL approximation [1]. The lines represent $r_{0} A^{1 / 3}$ dependence with $r_{0}$ deduced from the experimental radii of ${ }^{12} \mathrm{C}$ and ${ }^{16} \mathrm{O}$ given in Table $\mathrm{I}$.

by the two sets (HFB and HO) of the g.s. densities for $\mathrm{C}$ isotopes together with those deduced from the $\mathrm{OL}$ of Glauber model based on the same $\sigma_{\mathrm{I}}$ data [1]. One can see that our result follows closely the trend established by the OL of Glauber model, although the absolute $\left\langle r^{2}\right\rangle^{1 / 2}$ radii obtained with the HO densities are in most cases larger than those deduced from the OL of Glauber model. With the exception of the ${ }^{14} \mathrm{C}$ case, the radii of even $\mathrm{C}$ isotopes given by the microscopic $\mathrm{HFB}$ densities agree reasonably well with the empirical HO results. We have also plotted in Fig. 4 the lines representing $r_{0} A^{1 / 3}$ dependence with $r_{0}$ deduced from the experimental radii of ${ }^{12} \mathrm{C}$ and ${ }^{16} \mathrm{O}$ given in Table I. One can see that the behavior of nuclear radius in $\mathrm{C}$ isotopes is quite different from the $r_{0} A^{1 / 3}$ law. While $<r^{2}>^{1 / 2}$ radii found for ${ }^{12-15} \mathrm{C}$ agree fairly with the $r_{0} A^{1 / 3}$ law, those obtained for ${ }^{16-20} \mathrm{C}$ are significantly higher. In particular, a jump in the $\left\langle r^{2}\right\rangle^{1 / 2}$ value was found in ${ }^{16} \mathrm{C}$ compared to those found for ${ }^{12-15} \mathrm{C}$. This result seems to support the existence of a neutron halo in ${ }^{16} \mathrm{C}$ as suggested from the $\sigma_{\mathrm{R}}$ measurement for this isotope at $85 \mathrm{MeV} /$ nucleon [49]. We have further obtained a nuclear radius of 3.24 fm for ${ }^{19} \mathrm{C}$ which is significantly larger than that found for ${ }^{20} \mathrm{C}$. This result might also indicate to a neutron halo in this odd $\mathrm{C}$ isotope.

Situation is a bit different for $\mathrm{O}$ isotopes, where the best-fit $\left\langle r^{2}\right\rangle^{1 / 2}$ radii follow roughly the $r_{0} A^{1 / 3}$ law up to ${ }^{22} \mathrm{O}$. For the stable ${ }^{17,18} \mathrm{O}$ isotopes, the IPM densities [34] provide a very good description of the $\sigma_{\mathrm{I}}$ data (within
$1-2 \%)$. The best-fit HO densities give $<r^{2}>^{1 / 2}$ radii of 2.67 and $2.74 \mathrm{fm}$ for ${ }^{17} \mathrm{O}$ and ${ }^{18} \mathrm{O}$, respectively, which are rather close to those given by the IPM densities. Predictions given by the microscopic HFB densities are also in a good agreement with the data for even $\mathrm{O}$ isotopes excepting the ${ }^{24} \mathrm{O}$ case, where the HFB density gives obviously a too small $<r^{2}>^{1 / 2}$ radius. Since the HFB calculation already takes into account the continuum effects [33], such a deficiency might be due to the static deformation of ${ }^{24} \mathrm{O}$. A jump in the $\left\langle r^{2}\right\rangle^{1 / 2}$ value was found for ${ }^{23} \mathrm{O}$ which could indicate to a neutron halo in this isotope. Behavior of $<r^{2}>^{1 / 2}$ radii given by the bestfit HO densities agrees with the trend established by the OL of Glauber model [1] but, like the case of C isotopes, they are about $0.1 \mathrm{fm}$ larger than those deduced from the OL of Glauber model. Thus, the OL of Glauber model seems to consistently overestimate $\sigma_{\mathrm{R}}$ for the neutronrich $\mathrm{C}$ and $\mathrm{O}$ isotopes under study in comparison with our approach.

One clear reason for the difference between our results and those given by the OL of Glauber model analysis is that one has matched directly the calculated $\sigma_{\mathrm{R}}$ with the measured $\sigma_{\mathrm{I}}$ in the Glauber model analysis [1] to deduce the nuclear radius. If we proceed the same way with the $\mathrm{HO}$ densities for the considered nuclei, the best-fit $\left.<r^{2}\right\rangle^{1 / 2}$ radii decrease slightly but are still larger than those given by the OL of Glauber model. As already discussed in Sect. II, the zero-angle approximation for the NN scattering amplitude used in the Glauber model 
might reduce significantly the strength of the exchange part of the imaginary OP given by Eq. (12) and could overestimate, therefore, the absorption in the dinuclear system. This effect should be much stronger if one uses a realistic finite-range representation of the NN scattering amplitude. Bertsch et al. have shown [30] that the zerorange approximation for the $\mathrm{NN}$ scattering amplitude leads to a reduction of the calculated $\sigma_{\mathrm{R}}$ or an enhancement of the nuclear radius by a few percent (see Figs. 2 and 3 in Ref. [30]). Thanks to such a cancellation of the exchange effects by the zero-range approximation for NN scattering amplitude, the simple OL of Glauber model was able to deliver reasonable estimates of nuclear radii for many stable and unstable isotopes [1]. It should be noted that the eikonal approximation for the scattering wave function used in the Glauber model was introduced in the past to avoid large numerical calculations. With the computing power available today, there is no problem to perform the OM and DWIA calculations for different nucleus-nucleus systems involving large numbers of partial waves, and the folding + DWIA method presented here can be recommended as a reliable microscopic approach to predict the elastic scattering cross section and to deduce the nuclear radius from the measured $\sigma_{\mathrm{I}}$.

\section{CONCLUSION}

In this work we have explored the reliability of the optical model + DWIA approach as a tool for extracting important information on nuclear sizes from interaction cross section measurements. We concentrate on the energy region of 0.8 to $1 \mathrm{GeV} /$ nucleon where interaction cross section data exist for various combinations of stable as well as unstable projectiles on different targets. At these bombarding energies our knowledge of the empirical optical potential is scarce, especially for unstable systems, and we have used, therefore, the folding model to calculate the microscopic (complex) optical potential and inelastic form factors necessary for our analysis.

We have chosen for the folding input the fully finiterange $t$-matrix interaction developed by Franey and Love [13]. The folded optical potentials and inelastic form fac- tors are used further as inputs for the standard optical model and DWIA calculations of total reaction cross sections and interaction cross sections induced by stable and unstable $\mathrm{He}, \mathrm{Li}, \mathrm{C}$, and $\mathrm{O}$ isotopes on ${ }^{12} \mathrm{C}$ target. By using the well tested nuclear g.s. densities for the stable ${ }^{4} \mathrm{He},{ }^{12} \mathrm{C}$ and ${ }^{16} \mathrm{O}$ isotopes, we found that the Franey and Love $t$-matrix gives extremely good account of the measured $\sigma_{\text {I }}$ for these nuclei.

We have further used the nuclear g.s. densities obtained in various structure models to calculate $\sigma_{\mathrm{I}}$ and made realistic estimate for the nuclear radii of (still poorly known) neutron-rich isotopes based on the comparison between $\sigma_{\mathrm{I}}^{\text {calc }}$ and $\sigma_{\mathrm{I}}^{\text {exp }}$. For the chains of $\mathrm{C}$ and $\mathrm{O}$ isotopes, our results agree reasonably well with the empirical trend established by the OL of Glauber model [1], but give consistently larger $<r^{2}>^{1 / 2}$ radii for these nuclei. Such an effect could be due to the unsatisfactory treatment of the exchange part of the nucleus-nucleus OP in the Glauber model calculation.

Although the nuclear radii deduced by our approach for some light halo nuclei might be a few percent smaller than realistic values because the dynamic few-body correlation was not considered explicitly in the present folding + DWIA formalism, this fully microscopic approach was shown to be more accurate than the OL of Glauber model. Given realistic nuclear densities, it can give a reliable (parameter-free) prediction of the nucleus-nucleus optical potential at energies around $1 \mathrm{GeV} /$ nucleon. Therefore, it provides the necessary link to relate the calculated $\sigma_{\text {I }}$ to the nuclear density and RMS radius.

\section{Acknowledgments}

The authors thank G.R. Satchler for making the DOLFIN code available to them for the IPM calculation of nuclear g.s. densities, W. von Oertzen for helpful discussion, and W.G. Love for critical remarks to the manuscript. We also thank A. Ozawa, H. Sagawa, and I.J. Thompson for their correspondence on the nuclear densities. The research has been supported, in part, by the Natural Science Council of Vietnam.
[1] A. Ozawa, T. Suzuki, and I. Tanihata, Nucl. Phys. A693, 32 (2001).

[2] R.J. Glauber and G. Matthiae, Nucl. Phys. B21, 135 (1970).

[3] Y. Ogawa, K. Yabana, and Y. Suzuki, Nucl. Phys. A543, 722 (1992).

[4] I. Tanihata, J. Phys. G 22, 157 (1996).

[5] J.S. Al-Khalili, J.A. Tostevin, and I.J. Thompson, Phys. Rev. C 54, 1843 (1996).

[6] J.A. Tostevin, R.C. Johnson, and J.S. Al-Khalili, Nucl. Phys. A630, 340c (1998).

[7] G.R. Satchler and W.G. Love, Phys. Rep. 55, 183 (1979).
[8] Dao T. Khoa, G.R. Satchler, and W. von Oertzen, Phys. Rev. C 56, 954 (1997).

[9] G. Bertsch, J. Borysowicz, H. McManus, and W.G. Love, Nucl. Phys. A284, 399 (1977); N. Anantaraman, H. Toki, and G.F. Bertsch, Nucl. Phys. A398, 269 (1983).

[10] Dao T. Khoa and G.R. Satchler, Nucl. Phys. A668, 3 (2000).

[11] Dao T. Khoa, G.R. Satchler, and N.D. Thuy, Phys. Rev. C 65, 024611 (2002).

[12] W.G. Love and M.A. Franey, Phys. Rev. C 24, 1073 (1981)

[13] M.A. Franey and W.G. Love, Phys. Rev. C 31, 488 
(1985).

[14] G.R. Satchler, Direct Nuclear Reactions, Clarendon Press, Oxford, 1983.

[15] B. Sinha, Phys. Rep. 20, 1 (1975); B. Sinha and S.A. Moszkowski, Phys. Lett. 81B, 289 (1979).

[16] Dao T. Khoa, Phys. Rev. C 63, 034007 (2001).

[17] M.E. Farid and G.R. Satchler, Phys. Lett. B 146, 389 (1984).

[18] A. Bohr and B.R. Mottelson, Nuclear Structure (Benjamin, New York, 1975), Vol.2.

[19] A.K. Kerman, H. McManus, and R.M. Thaler, Ann. Phys. 8, 551 (1959).

[20] J. Jaros et al., Phys. Rev. C 18, 2273 (1978).

[21] Dao T. Khoa, G.R. Satchler, and W. von Oertzen, Phys. Lett. B 358, 14 (1995).

[22] A. Ozawa et al., Nucl. Phys. A691, 599 (2001).

[23] S. Raman, C. W. Nestor, Jr., and P. Tikkanen, At. Data and Nucl. Data Tables 78, 1 (2001).

[24] R.H. Spear, At. Data and Nucl. Data Tables 42, 55 (1989).

[25] J. Raynal, Computing as a Language of Physics (IAEA, Vienna, 1972) p.75; J. Raynal, coupled-channel code ECIS97 (unpublished).

[26] H. Esbensen and G.F. Bertsch, Phys. Rev. C 59, 3240 (1999).

[27] Dao T. Khoa and W. von Oertzen, Phys. Lett. B 304, 8 (1993); Dao T. Khoa and W. von Oertzen, Phys. Lett. B 342, 6 (1995).

[28] H. Nakada, Phys. Rev. C 68, 014316 (2003).

[29] M.S. Hussein, R.A. Rego, and C.A. Bertulani, Phys. Rep. 201, 279 (1991).

[30] G.F. Bertsch, B.A. Brown, and H. Sagawa, Phys. Rev.
C 39, 1154 (1989); H. Sagawa, Private communication (unpublished).

[31] M.E. Brandan, H. Chehime, and K.W. McVoy, Phys. Rev. C 55, 1353 (1997).

[32] Dao T. Khoa, G.R. Satchler, and W. von Oertzen, Phys. Rev. C 51, 2069 (1995).

[33] M. Grasso, N. Sandulescu, N. Van Giai, and R.J. Liotta, Phys. Rev. C 64, 064321 (2001).

[34] G.R. Satchler, Nucl. Phys. A329, 233 (1979).

[35] H. De Vries, C.W. De Jager, and C. De Vries, At. Data and Nucl. Data Tables 36, 503 (1987).

[36] V.B. Soubbotin and X. Viñas, J. Phys. G 25, 2087 (1999); Nucl. Phys. A665, 291 (2000).

[37] M.V. Zhukov, A.A. Korsheninnikov, and M.H. Smedberg, Phys. Rev. C 50, R1 (1994).

[38] G.D. Alkhazov et al., Nucl. Phys. A712, 269 (2002).

[39] J.S. Al-Khalili and J.A. Tostevin, Phys. Rev. C 57, 1846 (1998).

[40] V. Lapoux et al., Phys. Rev. C 66, 034608 (2002).

[41] I. Tanihata et al., Phys. Lett. B 206, 592 (1988).

[42] I. Tanihata et al., Phys. Rev. Lett. 55, 2676 (1985).

[43] T. Kobayashi et al., Phys. Lett. B 232, 51 (1989).

[44] K. Ikeda, T. Myo, and K. Kato, Nucl. Phys. A722, 335c (2003).

[45] T. Myo, S. Aoyama, K. Kato, and K. Ikeda, Phys. Lett. B 576, 281 (2003).

[46] E. Garrido, P. Sarriguren, E. Moya de Guerra, and P. Schuck, Phys. Rev. C 60, 064312 (1999).

[47] G.F. Bertsch and H. Esbensen, Ann. Phys. (N.Y.) 209, 327 (1991).

[48] S. Cohen and D. Kurath, Nucl. Phys. A101, 1 (1967).

[49] T. Zheng et al., Nucl. Phys. A709, 103 (2002). 